\title{
Computing with Daubechies' Wavelets
}

\author{
Adri B. Olde Daalhuis
}

\begin{abstract}
In the first two sections of this paper we describe a construction of the compactly supported Daubechies' wavelets. In the third section we show how a Discrete Wavelet Transform of a function can be obtained. We end this paper with some interesting numerical illustrations.
\end{abstract}

$\S 1$ Wavelets with finitely many non-zero filter coefficients In the theory of discrete wavelets the equation

$$
\phi(x)=\sqrt{2} \sum_{n=-\infty}^{\infty} h_{n} \phi(2 x-n)
$$

plays a fundamental role. With the farther wavelet $\phi$, satisfying this equation, we have the accompanying mother wavelet $\psi$ defined by

$$
\psi(x):=\sqrt{2} \cdot \sum_{n=-\infty}^{\infty}(-1)^{n} h_{1-n} \phi(2 x-n) .
$$

In Daubechies [1] for the first time a construction is given of wavelets resulting in compactly supported orthonormal wavelet bases. (See also Heijmans [this volume, $\S 4]$.)

It is not difficult to put conditions on $h_{n}$ of (1.1) in order to obtain compactly supported functions $\phi$ and $\psi$. We only need to have just a finite number of $\left\{h_{n}\right\}$ that are different from zero. In this section we give a proof of this property. 
We use the fact that the solution $\phi$ of equation (1.1) can be constructed by the following iteration scheme. Let

$$
\eta_{0}(x)= \begin{cases}1 & \text { as }-\frac{1}{2}<x<\frac{1}{2} \\ 0 & \text { elsewhere }\end{cases}
$$

That is, $\eta_{0}(x)$ is the characteristic function of $\left[-\frac{1}{2}, \frac{1}{2}\right]$. Next we define a sequence of functions $\eta_{l}, l=1,2, \ldots$, by writing

$$
\eta_{l}(x)=\sqrt{2} \sum_{n=-\infty}^{\infty} h_{n} \eta_{l-1}(2 x-n) .
$$

Then we have

$$
\phi(x)=\lim _{l \rightarrow \infty} \eta_{l}(x) .
$$

The reader is referred to [1] for a proof of this constructive procedure, which may also be used to draw pictures of solutions of (1.1).

Now, let just a finite number of filter coefficients $h_{n}$ of (1.1) be non-zero. That is, assume that we have two integer numbers $N_{-}, N_{+}$and that

$$
\begin{array}{ll}
h_{n}=0 & n<N_{-}, \\
& n>N_{+} .
\end{array}
$$

It is easily verified that the functions $\eta_{l}$ defined by (1.3) and (1.4) have compact support. We have in fact

$$
\operatorname{supp}\left(\eta_{l}\right)=\left[N_{l,-}, N_{l,+}\right]
$$

with

$$
\begin{array}{ll}
N_{0,-}=-\frac{1}{2}, & N_{0,+}=\frac{1}{2} \\
N_{l,-}=\frac{1}{2}\left(N_{l-1,-}+N_{-}\right), & N_{l,+}=\frac{1}{2}\left(N_{l-1,+}+N_{+}\right) .
\end{array}
$$

Thus we have

$$
\begin{aligned}
& N_{l,-} \rightarrow N_{-} \\
& N_{l,+} \rightarrow N_{+}
\end{aligned} \quad \text { as } l \rightarrow \infty,
$$

and it follows that

$$
\operatorname{supp}(\phi) \subset\left[N_{-}, N_{+}\right]
$$

and with (1.2)

$$
\operatorname{supp}(\psi) \subset\left[\frac{1}{2}\left(1-N_{+}+N_{-}\right), \frac{1}{2}\left(1+N_{+}-N_{-}\right)\right]
$$

In a recent paper of Lemarié-Rieusset \& Malgouyres [3], it is proven that the support of $\phi$ is indeed a connected interval. 
$\S 2$ Daubechies' construction of compactly supported wavelets

Daubechies' construction of compactly supported wavelets starts with the function

$$
H(\xi)=\frac{1}{\sqrt{2}} \sum_{n=-\infty}^{\infty} h_{n} e^{-i n \xi} .
$$

We prescribe that all $h_{n} \in \mathbb{R}$, and just a finite number are non-zero. The function $H(\xi)$ is of fundamental importance in the theory. It generates the function $\widehat{\phi}(\xi)$ :

$$
\widehat{\phi}(\xi)=\prod_{j=1}^{\infty} H\left(2^{-j} \xi\right)
$$

where the function $\widehat{\phi}(\xi)$ denotes the Fourier transform of the function $\phi(x)$ :

$$
\widehat{\phi}(\xi)=\int_{-\infty}^{\infty} e^{-i x \xi} \phi(x) d x
$$

So, when $H(\xi)$ is known, $\widehat{\phi}(\xi)$ and, hence, $\phi(x)$ can be constructed, and with (1.2) the mother wavelet $\psi(x)$ can be constructed.

The construction uses the following important theorem:

Theorem 1. (Daubechies) Let $h_{n}$ be a sequence such that

(i) $\sum_{n}\left|h_{n}\right||n|^{\varepsilon}<\infty$ for some $\varepsilon>0$,

(ii) $\sum_{n} h_{n-2 k} h_{n-2 l}=\delta_{k l}$,

(iii) $\sum_{n} h_{n}=\sqrt{2}$.

Suppose also that $H(\xi)=2^{-\frac{1}{2}} \sum_{n} h_{n} e^{-i n \xi}$ can be written as

$$
H(\xi)=\left[\frac{1}{2}\left(1+e^{-i \xi}\right)\right]^{N}\left[\sum_{n} f_{n} e^{-i n \xi}\right],
$$

where

(iv) $\sum_{n}\left|f_{n}\right||n|^{\varepsilon}<\infty$ for some $\varepsilon>0$,

(v) $\sup _{\xi \in \mathbb{R}}\left|\sum_{n} f_{n} e^{-i n \xi}\right|<2^{N-1}$

Define

$$
\begin{aligned}
g_{n} & =(-1)^{n} h_{1-n}, \\
\widehat{\phi}(\xi) & =\prod_{j=1}^{\infty} H\left(2^{-j} \xi\right), \\
\psi(x) & =\sqrt{2} \sum_{n} g_{n} \phi(2 x-n) .
\end{aligned}
$$


Then the $\phi_{j k}(x)=2^{-j / 2} \phi\left(2^{-j} x-k\right)$ define a multiresolution analysis, and the $\left\{\psi_{j k}\right\}$ are the associated orthonormal wavelet basis.

This theorem and the following theorems are proven in [1]. The number $N$ in (2.1) is crucial: when $N$ is large the wavelet has interesting properties with respect to approximations with the mother wavelets $\left\{\psi_{j k}\right\}$.

Remark. Starting with finitely many $h_{n}$, we obtain finitely many $f_{n}$, and $(i)$ and (iv) of Theorem 1 are obviously fulfilled.

Examples

$$
\begin{gathered}
h_{0}=h_{1}=\frac{1}{\sqrt{2}} \quad \text { and } \quad H(\xi)=\frac{1}{2}\left(1+e^{-i \xi}\right) . \\
h_{0}=\frac{1+\sqrt{3}}{4 \sqrt{2}}, h_{1}=\frac{3+\sqrt{3}}{4 \sqrt{2}}, h_{2}=\frac{3-\sqrt{3}}{4 \sqrt{2}}, h_{3}=\frac{1-\sqrt{3}}{4 \sqrt{2}} \\
H(\xi)=\left[\frac{1}{2}\left(1+e^{-i \xi}\right)\right]^{2} \frac{1}{2}\left[1+\sqrt{3}+(1-\sqrt{3}) e^{-i \xi}\right] .
\end{gathered}
$$

In the first example the function $\widehat{\phi}(\xi)$ can be computed easily. We have

$$
\widehat{\phi}(\xi)=\prod_{j=1}^{\infty} \frac{1}{2}\left(1+\exp \left(-i 2^{-j} \xi\right)\right)=\frac{1-e^{-i \xi}}{i \xi}
$$

Thus the father wavelet $\phi(x)$ and the associated mother wavelet $\psi(x)$ are the Haar wavelets:

$$
\phi(x)=\left\{\begin{array}{ll}
1 & \text { as } 0<x<1 \\
0 & \text { elsewhere }
\end{array} \quad \psi(x)= \begin{cases}1 & \text { as } 0<x<\frac{1}{2} \\
-1 & \text { as } \frac{1}{2}<x<1 \\
0 & \text { elsewhere. }\end{cases}\right.
$$

The first example is special with respect of symmetry because:

Theorem 2. (Daubechies) The Haar basis (associated to the above example (1)) is the only orthonormal basis of compactly supported wavelets for which the associated function $\phi$ has a symmetry axis.

The construction of the compactly supported wavelets from the function $H(\xi)$ starts by writing

$$
H(\xi)=\left[\frac{1}{2}\left(1+e^{-i \xi}\right)\right]^{N} Q\left(e^{-i \xi}\right) .
$$

The function $Q(x)$ is a polynomial with real coefficients. It is easy to see that $(i i)$ of Theorem 1 can be rewritten as

$$
|H(\xi)|^{2}+|H(\xi+\pi)|^{2}=1
$$


With (2.2) we have

$$
|H(\xi)|^{2}=\left[\cos ^{2} \frac{1}{2} \xi\right]^{N}\left|Q\left(e^{-i \xi}\right)\right|^{2} .
$$

Because of the coefficients of $Q(x)$ being real, we have $\overline{Q\left(e^{-i \xi}\right)}=Q\left(e^{i \xi}\right)$, and it follows that $\left|Q\left(e^{-i \xi}\right)\right|^{2}$ can be rewritten as a polynomial in $\cos \xi$, or, equivalently, as a polynomial in $\sin ^{2} \frac{1}{2} \xi$. So, with $y=\sin ^{2} \frac{1}{2} \xi$, there is a polynomial $P(y)$ such that

and

$$
P\left(\sin ^{2} \frac{1}{2} \xi\right)=\left|Q\left(e^{-i \xi}\right)\right|^{2},
$$

$$
P(y) \geq 0 \quad \forall y \in[0,1] .
$$

With this new polynomial, (2.3) becomes

$$
y^{N} P(1-y)+(1-y)^{N} P(y)=1 .
$$

Thus from a function $H(\xi)$, fulfilling (2.2) and (2.3), we obtain a polynomial $P(y)$, fulfilling (2.6) and (2.7). With the next Lemma it follows that from a polynomial $P(y)$, fulfilling (2.6) and (2.7), we can obtain a function $H(\xi)$, fulfilling (2.2) and (2.3), and this $H(\xi)$ has real $h_{n}$.

Lemma 1. (Riesz) Let $A(\xi)=\sum_{n=0}^{N} a_{n} \cos n \xi$, with $a_{n} \in \mathbb{R}$, be a positive trigonometric polynomial. Then there exists a trigonometric polynomial $B(\xi)=$ $\sum_{n=0}^{N} b_{n} e^{-i n \xi}$, with $b_{n} \in \mathbb{R}$, such that

$$
|B(\xi)|^{2}=A(\xi)
$$

The proof of this Lemma is given in [1], and it is constructive. We omit the proof.

A special solution of (2.6)-(2.7), which is given in [1], is

$$
P_{N}(y)=\sum_{j=0}^{N-1}\left(\begin{array}{c}
N-1+j \\
j
\end{array}\right) y^{j} .
$$

Because of all coefficients of $P_{N}(y)$ being positive, it fulfills (2.6), and the following relations, which were found by Tom Koornwinder, show that $P_{N}(y)$ fulfils (2.7).

$$
\begin{aligned}
& P_{N}(y)=N\left(\begin{array}{c}
2 N-1 \\
N
\end{array}\right)(1-y)^{-N} \int_{y}^{1} t^{N-1}(1-t)^{N-1} d t \\
& P_{N}(1-y)=N\left(\begin{array}{c}
2 N-1 \\
N
\end{array}\right) y^{-N} \int_{0}^{y} t^{N-1}(1-t)^{N-1} d t
\end{aligned}
$$

A general solution of (2.7) fulfils

$$
P(y)=(1-y)^{-N}-y^{N}(1-y)^{-N} P(1-y),
$$


thus $P_{N}(y)$ fulfils this relation, and this gives

$$
P(y)-P_{N}(y)=y^{N}(1-y)^{-N}\left[P_{N}(1-y)-P(1-y)\right] .
$$

So, a general solution of (2.7) is of the form

$$
P(y)=P_{N}(y)+y^{N} R(y)
$$

with $R(y)$ a polynomial that fulfils

$$
R(1-y)+R(y)=0 .
$$

Thus $P_{N}(y)$ is the polynomial-solution of (2.7) of minimal degree, and the polynomial $R(y)$ has to be antisymmetric with respect to $y=\frac{1}{2}$. Condition (2.6) gives some extra restrictions on $R(y)$.

To summarize, we have the following explicit characterization of all solutions $H(\xi)$ of (2.3), corresponding to only finitely many non-zero $h_{n}$. In the following theorem we have replaced $R$ by a polynomial which is antisymmetric with respect to $y=0$.

Theorem 3. Any trigonometric polynomial solution of (2.3) is of the form

$$
H(\xi)=\left[\frac{1}{2}\left(1+e^{-i \xi}\right)\right]^{N} Q\left(e^{-i \xi}\right),
$$

where $N \in \mathbb{N}, N \geq 1$, and where $Q$ is a polynomial such that

$$
\left|Q\left(e^{-i \xi}\right)\right|^{2}=\sum_{j=0}^{N-1}\left(\begin{array}{c}
N-1+j \\
j
\end{array}\right) \sin ^{2 j} \frac{1}{2} \xi+\left[\sin ^{2 N} \frac{1}{2} \xi\right] R\left(\frac{1}{2} \cos \xi\right)
$$

where $R$ is an odd polynomial, with some extra restrictions.

For obtaining the Daubechies' wavelets, we choose $R=0$. Thus for fixed $N$ the Daubechies' wavelets correspond to the trigonometric polynomials $H(\xi)$ of minimal degree. With this fixed $N$ we obtain from the polynomial $P_{N}(y)$ the polynomials $Q, H$, and the numbers $h_{n}$. Now we shall show that these $h_{n}, H$, and $Q$ fulfill the conditions of Theorem 1.

The conditions $(i)$ and $(i v)$ are obviously fulfilled, and (ii) is equivalent to (2.3), thus $(i i)$ is fulfilled. The special form $(2.2)$ of $H(\xi)$ gives that $H(\pi)=0$. Thus with (2.3) we have $1=|H(0)|^{2}=\frac{1}{2}\left(\sum h_{n}\right)^{2}$, and (iii) is fulfilled.

Condition $(v)$ is $\left|Q\left(e^{-i \xi}\right)\right|<2^{N-1}$, and it is fulfilled because of

$$
\left|Q\left(e^{-i \xi}\right)\right|^{2}=P_{N}\left(\sin ^{2} \frac{1}{2} \xi\right) \leq P_{N}(1)=\left(\begin{array}{c}
2 N-1 \\
N
\end{array}\right)<2^{2(N-1)}
$$


In the construction (via the proof of Lemma 1) of $Q$ from $|Q|^{2}$, we obtain all zeros of $Q$ inside the unit circle, and this determines $Q$ unambiguously, up to a phase factor $e^{i K \xi}, K \in \mathbb{Z}$. We fix this phase factor so that $Q$ contains only negative frequencies, starting from zero, i.e.,

$$
Q_{N}\left(e^{-i \xi}\right)=\sum_{n=0}^{N-1} q_{n} e^{-i n \xi} \quad \text { with } \quad q_{0} \neq 0 .
$$

\section{Examples}

$$
\begin{gathered}
Q_{2}(\xi)=\frac{1}{2}\left[1+\sqrt{3}+(1-\sqrt{3}) e^{-i \xi}\right] \\
Q_{3}(\xi)=\frac{1}{4}\left[1+\sqrt{10}+\sqrt{5+2 \sqrt{10}}+2(1-\sqrt{10}) e^{-i \xi}\right. \\
\left.+(1+\sqrt{10}-\sqrt{5+2 \sqrt{10}}) e^{-2 i \xi}\right]
\end{gathered}
$$

With this $Q_{N}$ we have

$$
\begin{aligned}
H_{N}(\xi) & =\left[\frac{1}{2}\left(1+e^{-i \xi}\right)\right]^{N} \sum_{n=0}^{N-1} q_{n} e^{-i n \xi} \\
& =2^{-\frac{1}{2}} \sum_{n=0}^{2 N-1} h_{n} e^{-i n \xi} .
\end{aligned}
$$

Let us denote the corresponding $\phi, \psi$ functions by $\phi_{N}, \psi_{N}$. The theory of Section 1 gives

$$
\operatorname{supp}\left(\phi_{N}\right)=[0,2 N-1], \quad \operatorname{supp}\left(\psi_{N}\right)=[-N+1, N]
$$

For $N=2$ and $N=3$ the associated $h_{n}$ can be calculated exactly, and for $N \leq 10$ Daubechies gives the numerical values of the $h_{n}$ in [1].

When $N$ increases with 1 , the number of non-zero $h_{n}$ increases with 2 . And when $N$ increases the smoothness of $\phi_{N}$ and $\psi_{N}$ increases:

Theorem 4. (Daubechies) There exists $\lambda>0$ such that, for all $N \in \mathbb{N}, N \geq 2$,

$$
\phi_{N}, \psi_{N} \in C^{\lambda N} \text {. }
$$

In Daubechies [2, p. 226] it is proven that for large $N$ the optimal $\lambda$ is

$$
\lambda=1-\frac{\ln 3}{\ln 4} \sim 0.2075 \text {. }
$$


$\S 3$ Computing with the compactly supported Daubechies' wavelets

In this section we show how a Discrete Wavelet Transform of a function can be obtained. We will use the Daubechies' wavelet $\phi_{N}$ of the previous section, and, again, the associated functions are $\phi_{j k}(x)=2^{-j / 2} \phi_{N}\left(2^{-j} x-k\right)$. A function will be represented by a finite signal $\bar{a}=\left(a_{0}, \cdots, a_{M-1}\right)^{T}$. As an intermediate expression we introduce $A=\sum_{j=0}^{M-1} a_{j} \phi_{K j}$, with $M=2^{K}$. Then the Discrete Wavelet Transform of $\bar{a}$ appears to be constituted by $2^{K}-2$ coefficients of the expansion of $A$ in the orthonormal $\psi_{n m}$ basis.

We choose $N$ fixed, and with the filter-coefficients $h_{n}$ of $\phi_{N}$ we define filters $\mathbf{H}_{N}, \mathbf{G}_{N}: l^{2} \rightarrow l^{2}$

$$
\begin{aligned}
\left(\mathbf{H}_{N} \bar{a}\right)_{k} & =\sum_{l=-\infty}^{\infty} h_{l-2 k} a_{l}, \\
\left(\mathbf{G}_{N} \bar{a}\right)_{k} & =\sum_{l=-\infty}^{\infty} g_{l-2 k} a_{l},
\end{aligned}
$$

with again $g_{n}=(-1)^{n} h_{1-n}$.

Now we let these filters work on the finite signal $\bar{a}=\left(a_{0}, \cdots, a_{M-1}\right)^{T}$, with $M$ even. In matrix-form $\mathbf{H}_{N}$ is

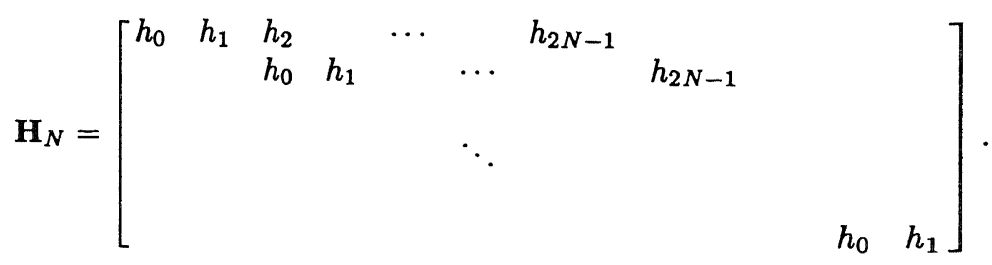

The matrix-form of $\mathrm{G}_{N}$ is the same, with $h_{n}$ replaced by $g_{n}$. Filtering with these $\frac{1}{2} M \times M$-matrices will cause edge effects for $N>1$. For eliminating these edge effects, we make these matrices periodic in the following way

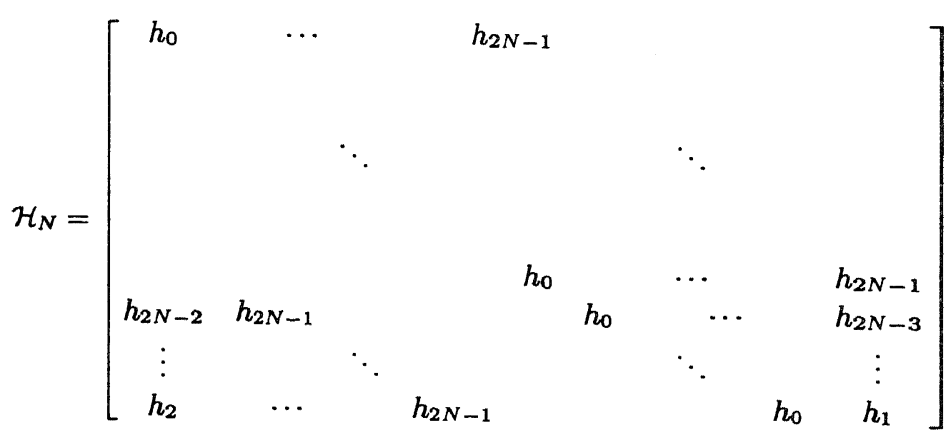


with $\mathcal{G}_{N}$ the same as $\mathcal{H}_{N}$ with $h_{n}$ replaced by $g_{n}$. This is the same as leaving the filters $\mathbf{H}_{N}$ and $\mathbf{G}_{N}$ unchanged and making the signal $\bar{a}$ periodic to a $l^{\infty}$-vector.

The total filtering is now

$$
\left[\begin{array}{c}
\mathcal{H}_{N} \\
\mathcal{G}_{N}
\end{array}\right]_{M}\left(\begin{array}{c}
a_{0} \\
\vdots \\
a_{M-1}
\end{array}\right)=\left(\begin{array}{c}
\mathcal{H}_{N}\left(\begin{array}{c}
a_{0} \\
\vdots \\
a_{M-1}
\end{array}\right) \\
\mathcal{G}_{N}\left(\begin{array}{c}
a_{0} \\
\vdots \\
a_{M-1}
\end{array}\right)
\end{array}\right): \mathbb{R}^{M} \rightarrow \mathbb{R}^{M}
$$

The matrix $\left[\begin{array}{c}\mathcal{H}_{N} \\ \mathcal{G}_{N}\end{array}\right]_{M}$ is orthonormal, i.e.,

$$
\left[\begin{array}{c}
\mathcal{H}_{N} \\
\mathcal{G}_{N}
\end{array}\right]_{M}\left[\mathcal{H}_{N}^{T} \mathcal{G}_{N}^{T}\right]_{M}=\left[\begin{array}{cc}
\mathcal{H}_{N} \mathcal{H}_{N}^{T} & \mathcal{H}_{N} \mathcal{G}_{N}^{T} \\
\mathcal{G}_{N} \mathcal{H}_{N}^{T} & \mathcal{G}_{N} \mathcal{G}_{N}^{T}
\end{array}\right]_{M}=I d_{M}
$$

This follows directly from the equations

$$
\begin{gathered}
\sum_{n=-\infty}^{\infty} h_{n-2 k} h_{n-2 l}=\delta_{k l} \\
\sum_{n=-\infty}^{\infty} h_{n-2 k} g_{n-2 l}=0
\end{gathered}
$$

where (3.7) is condition (ii) of Theorem 1, and (3.8) follows from

$$
\left.\sum_{n=-\infty}^{\infty} h_{n-2 k} g_{n-2 l}=\sum_{n=-\infty}^{\infty}<\phi_{1 k}, \phi_{0 n}><\phi_{0 n}, \psi_{1 l}>=<\phi_{1 k}, \psi_{1 l}\right\rangle=0
$$

We define

$$
\phi_{m n}(x)=2^{-\frac{m}{2}} \phi\left(2^{-m} x-n\right), \quad \psi_{m n}(x)=2^{-\frac{m}{2}} \psi\left(2^{-m} x-n\right),
$$

and we choose $M=2^{K}, K \in \mathbb{N}^{*}$. Let $A \in V_{K}$ such that

$$
a_{k}=a_{k 0}=<A, \phi_{K k}>\text {. }
$$

Then we define

$$
a_{k j}=<A, \phi_{K-j, k}>, \quad d_{k j}=<A, \psi_{K-j, k}>.
$$


The Discrete Wavelet Transformation becomes (for $K=4$ )

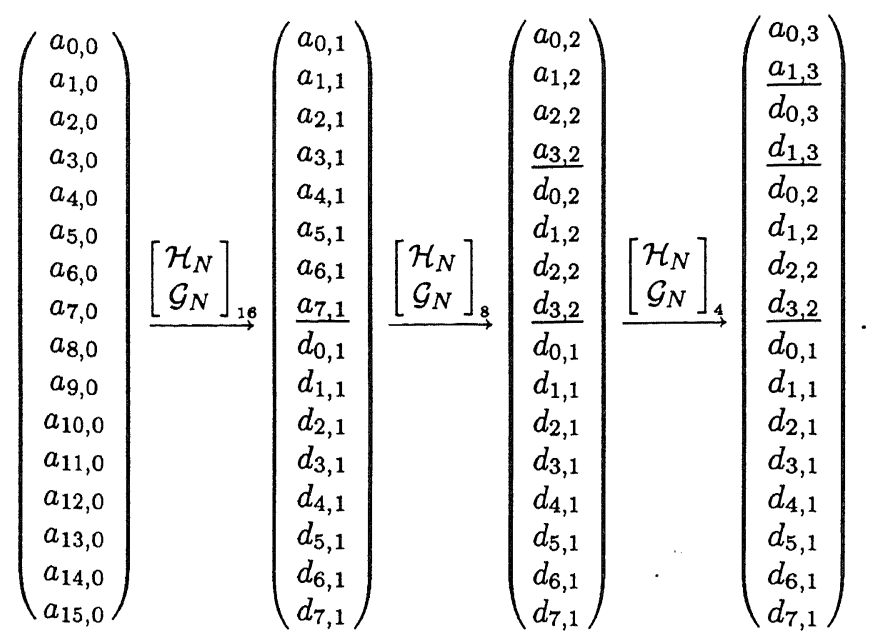

So, for general $K$, the Discrete Wavelet Transformation is built up by simple matrix-operations with orthonormal matrices. The special form of these matrices make these matrix-operations, and the Discrete Wavelet Transform for general $K$, easy to program. And the whole process is simple to invert. Notice that the transformation is a process that transforms a signal of length $2^{K}$ into a vector of length $2^{K}$.

The $d_{j k}$ are some of the coefficients of the expansion of $A$ in the orthonormal $\psi_{j k}$ basis. The remaining $a_{0, K-1}$ and $a_{1, K-1}$ are called the "mother-function coefficients".

\section{$\S 4$ What do Daubechies' wavelets look like, and how do they work on signals?}

The illustrations in this section are made on a Macintosh-II using a Pascal program, which is based on the program given in Press [4]. In the illustrations, the length of the signals $M=1024$. First we show some figures of the $\phi_{N}$ and $\psi_{N}$ for some different values of $N$. Notice that a signal of $\psi_{N}$ can be obtained by starting at the right hand side of scheme (3.12) with a vector $\delta_{i}=\left\{\delta_{i k}\right\}_{k=1}^{1024}$, where $i \in 2, \cdots, 1024$ is fixed. This follows directly from (3.11). The figures are shown in Figures 1, 2, and 3.

Notice that, according to Theorems 2 and 4 , the smoothness of the wavelets increases with $N$, and only for $N=1$ the $\phi_{N}$ has a symmetry axis. The inverse discrete wavelet transform of $\delta_{10}+\delta_{58}$ is shown in Figure 4. 


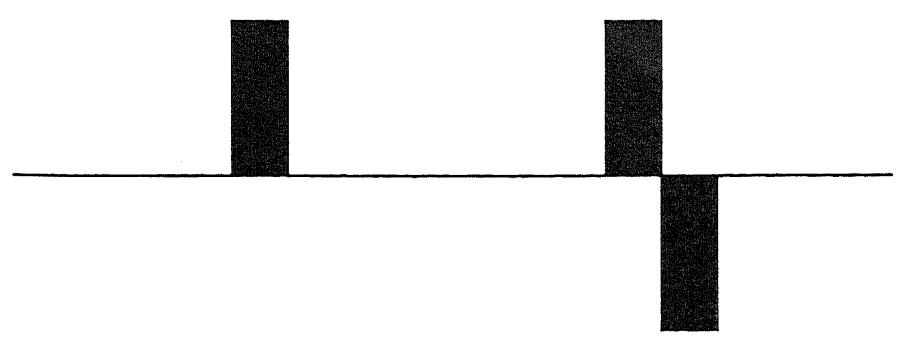

Figure 1. Father (left) and mother wavelet for $N=1$, the Haar functions.

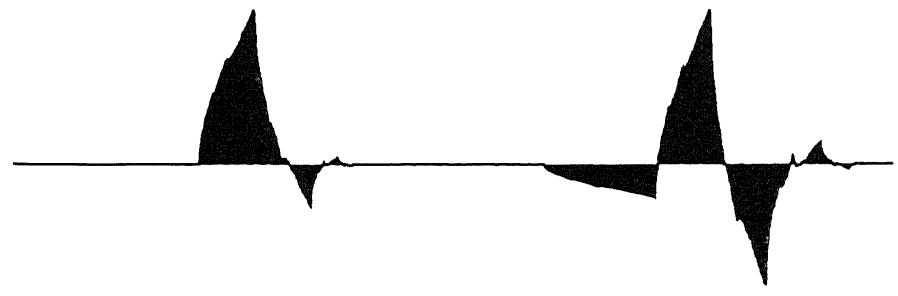

Figure 2. Father (left) and mother wavelet for $N=2$.

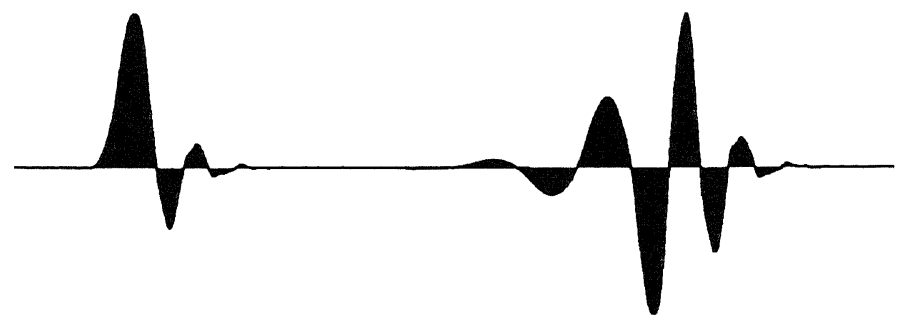

Figure 3. Father (left) and mother wavelet for $N=6$.

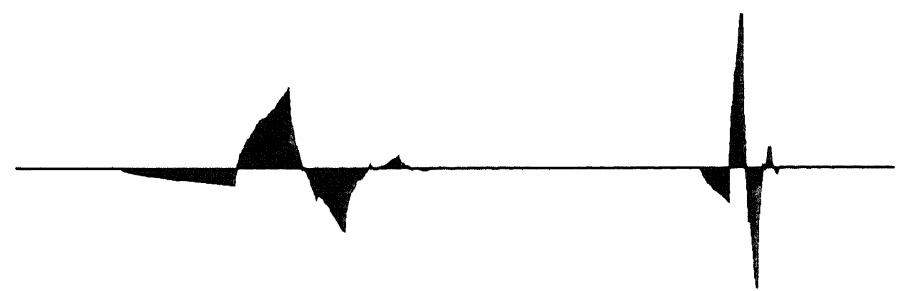

Figure 4. The inverse discrete wavelet transform of $\delta_{10}+\delta_{58}$ for $N=2$. 


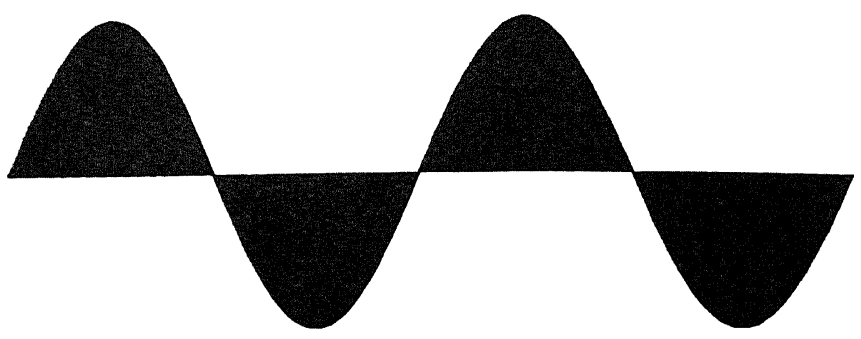

Figure 5. The sinus-signal.

Since 10 lies early in the hierarchical range 9-16, that wavelet lies on the left side of the picture. Since 58 lies in a later (smaller scale) hierarchy, it is a narrower wavelet.

The discrete wavelet transforms of the sinus-signal (see Figure 5) are calculated for $N=1$ and $N=10$. They are shown in Figures 6 and 7 .

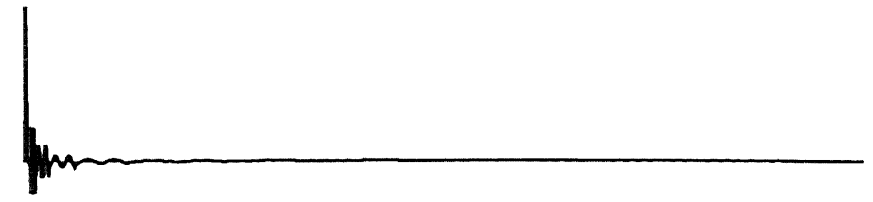

Figure 6. Discrete wavelet transform of the sinus-signal for $N=1$.

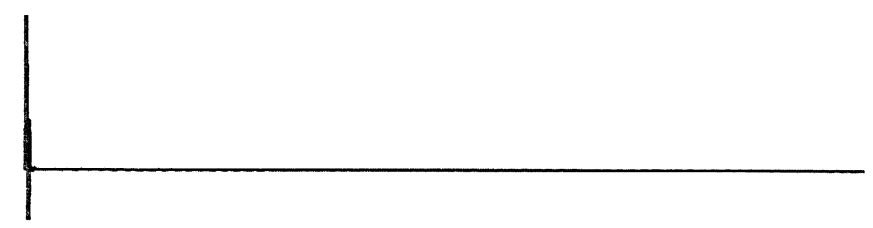

Figure 7. Discrete wavelet transform of the sinus-signal for $N=10$.

The amplitudes of the transform for $N=10$ are more concentrated at the left part of the signal then the amplitudes of the transform for $N=1$. This can be explained by the smoothness of the underlying wavelets. The wavelets for $N=10$ match better to the smooth signal. In other cases, when the original signal is more singular, the wavelets for $N=1$ match better to that signal.

Now we can truncate these wavelet transforms. For $N=1$ there are 18 coefficients that have amplitudes larger then 0.05 times the maximum amplitude of the transform. We set the remaining amplitudes to zero. With this kind of datareduction we have to record both the values and the positions of the non-zero 
coefficients. Thus in the case of $N=1$ we reduce to a vector of length 36 . And in the case of $N=10$ we reduce to a vector of length 12 . The following two pictures show the result of truncations of the original signal from the two inverse discrete wavelet transforms of the truncated vectors.

\section{W-Astrom}

Figure 8. Original signal minus approximation signal for $N=1$.

Figure 9. Original signal minus approximation signal for $N=10$.

So, when we start with a smooth signal, the data-reduction with $N=10$ is much better.

Remark. It is very important that vectors in wavelet space be truncated according to the amplitude of the components, not their position in the vector. Keeping the first 16 components of the vector would give an extremely poor approximation to the original signal.

\section{References}

1. Daubechies, I., Orthonormal bases of compactly supported wavelets, Comm. Pure and Appl. Math. 41 (1988), 909-996.

2. Daubechies, I., Ten Lectures on Wavelets, CBMS-NSF, Regional Conference Series in Applied Mathematics \# 61, SIAM, Philadelphia, Pennsylvania, 1992.

3. Lemarié-Rieusset, P.G. and G. Malgouyres, Support des fonctions de base dans une analyse multi-résolution, C. R. Acad. Sci. Paris Sér. I Math. 313 (1991), 377-380.

4. Press, W.H., Wavelet transforms, Harvard-Smithsonian Center for Astrophysics, No. 3184, 1991, preprint.

Adri B. Olde Daalhuis

CWI

P.O. Box 4079

1009 AB Amsterdam

The Netherlands

aod@cwi.nl 